DOI 10.26886/2523-6938.1(2)2018.9

UDC: 617.3

ANALYSIS OF THE DYNAMICS OF CHANGING THE INDICATORS OF

THE FUNCTIONAL OCCLUSION ASSESSMENT METHOD

T-SCAN IN PATIENTS WITH OCCLUSION DISORDERS WHICH HAVE BEEN ARISEN OR WERE ACTIVATED AS A RESULT OF INCORRECT

\title{
DENTAL SURGERY
}

\section{A. Shynchukovskyi, PhD, Associate Professor, \\ V. P. Nespryadko, MD, PhD, Professor, \\ O. G. Tereshchuk, PhD, Assistant, \\ N. S. Khrol, PhD, Associate Professor,}

\section{A. V. Oleinyk}

A. A. Bogomolets National Medical University, Ukraine, Kiev

Recent studies showed that regaining the occlusal contacts is one of the most difficult tasks during the prosthetics remanufacturing process. It often happens, that during prosthetics remanufacturing process misunderstanding between a patient and a doctor may occur. It is related to the removal of expensive structures and manufacturing of new ones that are just as pricey. Thus, the touchstone for achieving optimal functional and esthetic results is not only the repositioning of the mandible that eliminates pain sensation in TMJ, but also regaining of the occlusal contacts is needed for prosthetics remanufacturing. Thereby, by knowing the treatment outcome, the doctor is able to minimize the risk of error in prosthetics remanufacturing process and improve the doctor-patient relationship.

Key words: maxillo-dental apparatus, temporomandibular joint, occlusive correction, masticatory muscles, pharmaceutical constructions

Summary. We brought to the light that one of the the most controversial issues is the moment of adaptation to reconstructive procedures. Often it becomes unclear which and in which order to use 
medical diagnostic equipment, to training for fixed prosthetic stages. Our studies make it possible to predict not only the treatment time, but the amount of occlusal adjustment in conjunction with the reconstruction of the position of the mandible.

One of the most debated issues is the time of adaptation to reconstructive manipulation. Often it becomes unclear how and in what order medical diagnostic equipment should be used in the preparatory stages of the stationary prosthetics. Our studies make it possible to predict not only the treatment time, and the amount of occlusal adjustment in conjunction with the reconstruction of the lower jaw.

Work objective: optimization of the diagnostic and treatment process by analyzing the dynamics of changes in T-scan indicators, which will minimize the risk of error in carrying out re-prosthetics after removal of the structures that have become a trigger factor or the cause of manifestations of functional disorders of dento-maxillofacial apparatus.

Introduction. Any occlusive disorders, in particular those that have arisen or were activated as a result of orthopedic treatment, as well as partial loss of teeth, are accompanied by changes in almost all areas of the dento-maxillofacial apparatus(DMFA) and lead to a number of complications. The lower jaw with a complex of muscles of mastication, temporomandibular joint (TMJ), dentitions are an integral part of the permanent maxillo-facial area, which provides the primary processing of food and other vital functions of the human body. The emergence of disturbed occlusion and pathologic behaviour in the temporomandibular joint are affected by a variety of factors. These factors include somatic diseases and injuries of the maxillo-facial area, the age-related status of dentitions and occlusion, the presence of tooth fillings and defects in the dentitions, the quality of dental prostheses in the oral cavity, the pathological abrasion of hard tissues of the teeth, bad habits etc. 
This kind of morphological changes significantly complicate orthopedic treatment of functional disorders of dento-maxillofacial apparatus, especially if they are determined by somatic pathology. Sometimes such treatment becomes impossible without the use of a set of special preparatory measures (Gross M. D., Mathews J. D, 1986; Timofeev, A. A. Mirza A. I., 2001; Mirza A. I. 2002; Rozhko M. M., Herzanych N. I., 2008; Riabokon E. N., 2008; Khaibulina R. R., 2008; MD Gross, JD Mathews, 1982; L. Helenius, 2005).

Many people have dentitions with some elements of occlusal disharmony, which leads to the development of "muscle stress". In this case, the masticatory system loses its ability to adapt adequately, the functional state of the TMJ elements is disrupted, with the result that inflammatory and dystrophic changes may occur.

In most cases, these factors are unstable and under a loss of adaptive capacity, they can lead to dysfunction of the elements of dentomaxillofacial apparatus, in particular, elements of TMJ, and acquire temporary or chronic forms [3, 4].

The mere list of typical primary symptoms in dysfunction shows that it is due to the state of the muscles and occlusion, which in turn ensure the condition and function of the joint. Therefore, the diagnosis of "arthritis" or "arthrosis", which is often established in these cases, should have a clear pathogenetic basis, which requires the use of a comprehensive study of the organs and structures of the maxillo-facial area [2, 3, 4].

If the loss of a significant amount of teeth occurred, the antagonizing teeth are displaced, the contact points on some teeth disappear, the teeth are placed fanwise, which leads to a decrease in the occlusal vertical dimension. Alveolar processes atrophy, and the patient has to push the lower jaw forward when chewing a food. A decrease in the occlusal vertical dimension leads to a functional displacement of the 
masticatory apparatus together with changes in the TMJ and masticatory muscles. Recovering the vertical dimension and the position of the lower jaw as they werebefore any changes in the dentitions means that the masticatory apparatus must be secondly replaced, which is undesirable, and sometimes impossible, due to profound anatomic changes in the TMJ. If the period after tooth loss is long enough, the compensatory-adaptive position of the lower jaw becomes stable, which can lead to misdiagnosing in determining the central ratio of the jaws. Difficulties also arise because the markings, which make it possible to restore the occlusal vertical dimension, are lost as a result of the loss of teeth and atrophy of the alveolar processes.

It should be noted that the nature of the occlusion of teeth is affected by "factors of occlusion", such as: morphological structure of the posterior tooth occlusal surface, incisor path, terminal hinge axis, compensatory curves, articular sagittal and transversal pathways and angles. That is why any indirect restorative dental manipulation requires careful analysis of the ratio between the upper and lower jaws at the time of the re-prosthetics.

Materials and methods: the work is considering the application of various approaches to the analysis of occlusal adjustment of dentitions in the preparatory re-prosthetics stage. Therefore, our patients were allocated to treatment groups.

In total, we examined and treated 134 (100\%) patients First group of 60 patients $(44.8 \%)$ - with multiple direct and indirect orthopedic constructions of small and medium length Second group of 40 of patients (29.9\%) - with complete orthopedic treatment with removable and non-removable structures

First subgroup of the second group of 23 patients (17.2\%, 57.5\%) - with full preliminary prosthetics with non-removable ceramic structures with pronounced occlusion surface architectonics 
Second subgroup of the second group of 17 patients $(12.7 \%, 42.5 \%)$ with full preliminary prosthetics with removable and non-removable ceramic structures with implied occlusal surface architectonics in the areas Third group of 34 patients (25.4\%) - they are different after dental status in both I and II groups, but they have a general somatic pathology that became the primary cause in the development of neurotic symptoms.

Discussion. The above changes in the indicators of functional analysis of occlusal adjustments using the T-scan method are associated not only with the dental status, but also with the dominance of certain symptomatic manifestations in each group. The distribution of symptomatic manifestations is as follows. First group-muscle symptoms (with painful components), audible manifestations in TMJ, sense of discomfort by disocclusion. Second group - to the symptoms of the first group, a pain in TMJ area and difficult opening of mouth are added. Third group - to the symptoms of first and second groups, the neurotic symptoms are added in the form of irradiation of pain along the branches of the trigeminal nerve and the presence of trigger points.

In the first group of patients - $60(44.8 \%)$ patients with multiple direct and indirect orthopedic constructions of small and medium length, we observed muscular symptoms without pain from TMJ area.

In the patients of this clinical group, clinical benefits were observed immediately after the prosthetics was performed, and the stabilization of these indicators 3 months after the treatment can be explained by the intensity of the muscular component in the disease process in patients with this dental status, as the dominants in its occurrence. These dental rebuilding were carried out within different time periods, which led to persistent functional changes in the operation of the elements of dentomaxillofacial apparatus. 
In the second group of 40 patients (29.9\%) with complete orthopedic treatment with removable and non-removable structures, we observed muscular symptoms with pain from the TMJ area.

Clinical benefits: the clinical benefits were observed immediately after the treatment, as the prosthetics were carried out simultaneously and the change in the ratio occurred quickly and in the same time period.

In the third group of 34 patients $(25.4 \%)$ with different dental status in both I and II groups, we have observed a general somatic pathology that became the primary cause in the development of neurotic symptoms. We have observed improvement and stabilization of indicators at later stages of treatment, which was directly proportional to the treatment of specialists in related branches.

After the treatment, we conducted a second study in all groups immediately after treatment, and 3 and 6 months after treatment. The results of the dynamic changes in the values of functional analysis of occlusal adjustments using the T-scan method are given in Tables 2-4.

Table 2

Dynamic changes for the first clinical group

\begin{tabular}{|l|c|c|c|c|}
\hline \multicolumn{1}{|c|}{ Parameter } & Beforedentalprosthetics & $\begin{array}{c}\text { After } \\
\text { dentalprosthetics }\end{array}$ & $\begin{array}{c}\text { In } \\
3 \text { months }\end{array}$ & $\begin{array}{c}\text { In } \\
6 \text { months }\end{array}$ \\
\hline Occlusiontime & Upto $0.52 \mathrm{~ms}$ & Upto $0.41 \mathrm{~ms}$ & $\begin{array}{c}\text { Upto } \\
0.32 \mathrm{~ms}\end{array}$ & $\begin{array}{c}\text { Upto } \\
0.3 \mathrm{~ms}\end{array}$ \\
\hline Disocclusion time & Upto $0.7 \mathrm{~ms}$ & Upto $0.5 \mathrm{~ms}$ & Upto & Upto \\
& & & $0.38 \mathrm{~ms}$ & $0.35 \mathrm{~ms}$ \\
\hline $\begin{array}{l}\text { Balance of the right and } \\
\text { left side }\end{array}$ & Upto 20\%-80\% & $45 \%-55 \%$ & $45 \%-55 \%$ & $45 \%-55 \%$ \\
\hline
\end{tabular}


Table 3

Dynamic changes for the second clinical group

\begin{tabular}{|c|c|c|c|c|}
\hline Parameter & Beforedentalprosthetics & $\begin{array}{c}\text { After } \\
\text { dentalprosthetics }\end{array}$ & $\begin{array}{c}\text { In } \\
3 \text { months }\end{array}$ & $\begin{array}{c}\text { In } \\
6 \text { months }\end{array}$ \\
\hline Occlusiontime & Upto $0,2 \mathrm{~ms}$ & Upto $0,25 \mathrm{~ms}$ & $\begin{array}{c}\text { Upto } \\
0,23 \mathrm{~ms}\end{array}$ & $\begin{array}{c}\text { Upto } \\
0,25 \mathrm{~ms}\end{array}$ \\
\hline Disocclusion time & Upto $0,15 \mathrm{~ms}$ & Upto $0,30 \mathrm{~ms}$ & $\begin{array}{c}\text { Upto } \\
0,27 \mathrm{~ms}\end{array}$ & $\begin{array}{c}\text { Upto } \\
0,26 \mathrm{~ms}\end{array}$ \\
\hline $\begin{array}{l}\text { Balance of the right and } \\
\text { left side }\end{array}$ & $\begin{array}{l}\text { Up to } 45 \%-55 \% \\
\text { (first subgroup) } \\
\text { Up to } 70 \%-30 \% \\
\text { (secondsubgroup) }\end{array}$ & $\begin{array}{l}\text { Upto } 50 \%-50 \% \\
\text { Upto } 45 \%-55 \%\end{array}$ & $\begin{array}{l}\text { Upto } 45 \%- \\
55 \% \\
\text { Upto } 45 \%- \\
55 \%\end{array}$ & $\begin{array}{l}\text { Upto } 45 \%- \\
55 \% \\
\text { Upto } 45 \%- \\
55 \%\end{array}$ \\
\hline
\end{tabular}

Table 4

\section{Dynamic changes for the third clinical group}

\begin{tabular}{|l|c|c|c|c|}
\hline \multicolumn{1}{|c|}{ Parameter } & Beforedentalprosthetics & $\begin{array}{c}\text { After } \\
\text { dentalprosthetics }\end{array}$ & $\begin{array}{c}\text { In } \\
3 \text { months }\end{array}$ & $\begin{array}{c}\text { In } \\
6 \text { months }\end{array}$ \\
\hline Occlusiontime & Upto $0,70 \mathrm{~ms}$ & Upto $0,42 \mathrm{~ms}$ & Upto \\
& & & Upto \\
& & Upto $0,30 \mathrm{~ms}$ & $0,50 \mathrm{~ms}$ \\
\hline Disocclusion time & Upto $0,30 \mathrm{~ms}$ & Upto & Upto \\
& & & $0,26 \mathrm{~ms}$ & $0,26 \mathrm{~ms}$ \\
\hline Balance of the right and & Upto 40\%-60\% & Upto 50\%-50\% & Upto $45 \%-$ & Upto $45 \%-$ \\
left side & & & $55 \%$ & $55 \%$ \\
\hline
\end{tabular}

For this reason, when the question is about prosthetics, the following factors should be taken into account:

- Symptomatic manifestations

- Dental status

- Difference in the implementation of dental restorations in a temporal aspect. 
Results: Such allocation of patients to treatment group allowed us to analyzethe results of treatment in the prognostic aspect. After all, it allowed giving an opinion on the planning of further treatment.

The results of first group of 60 patients $(44.8 \%)$ are shown in Fig. 1, 2. Occlusion time indicators are increased to $0.52 \mathrm{~ms}$, the disocclusion time is increasedto $0.70 \mathrm{~ms}$, the balance of the right and left side is $20 \%-80 \%$, that is $60 \%-80 \%$ of the allowable limit, which is due to the unpronounced occlusal surface architectonics of the teeth and dentitions and the possibility of occlusion in different positions.

The results of second group of 40 patients (29.9\%) are shown in Fig. 3, Table 1. (for both subgroups). Occlusion time indicators are decreased to $0.2 \mathrm{~ms}$, the disocclusion time is decreased to $0.1 \mathrm{~ms}$.

In the first subgroup of the second group of 23 patients $(17.2 \%$, $57.5 \%$ ), the balance of the right and left side is $45 \%-55 \%$, which corresponds to the norm.

This is due to the pronounced occlusal surface architectonics of the teeth and dentitions and the possibility of occlusion in one position, which can be explained by the pronouncement of the steepness and height of the slopes of the dental tubercles.

In the second subgroup of the second group of 17 patients $(12.7 \%$, $42.5 \%$ ), the balance of the right and left side is $70 \%-30 \%$, that is $60 \%$ $80 \%$ of the allowable limit. This is due to impaired fixation and improperly restored distal support on removable previous orthopedic structures.

\begin{tabular}{|l|c|}
\hline \multicolumn{1}{|c|}{ Parameter } & Beforetreatment \\
\hline Occlusiontime & Upto $0.52 \mathrm{~ms}$ \\
\hline Disocclusion time & Upto $0,70 \mathrm{~ms}$ \\
\hline Balance of the right and left side & Upto $20 \%-80 \%$ \\
\hline
\end{tabular}




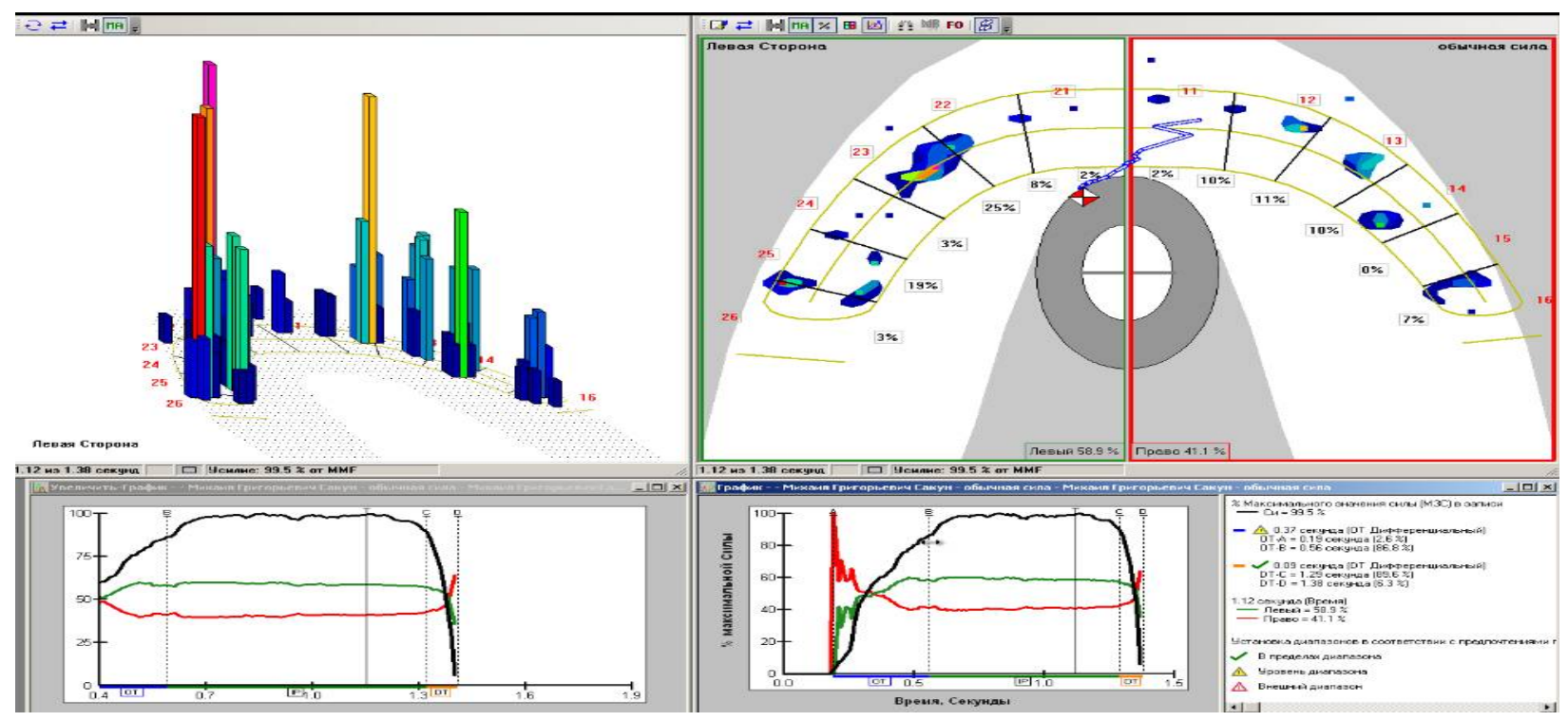

Fig. 1

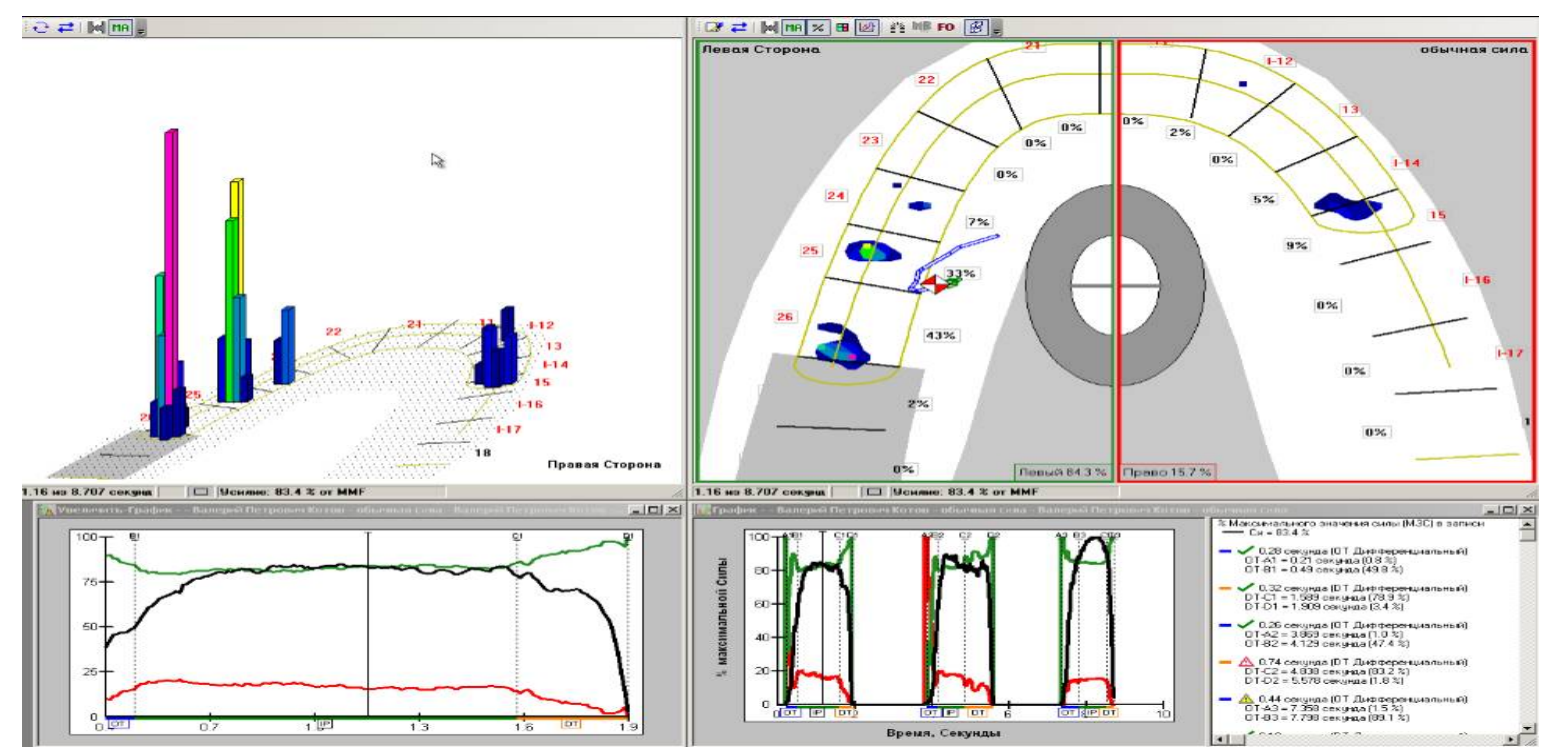

Fig. 2 


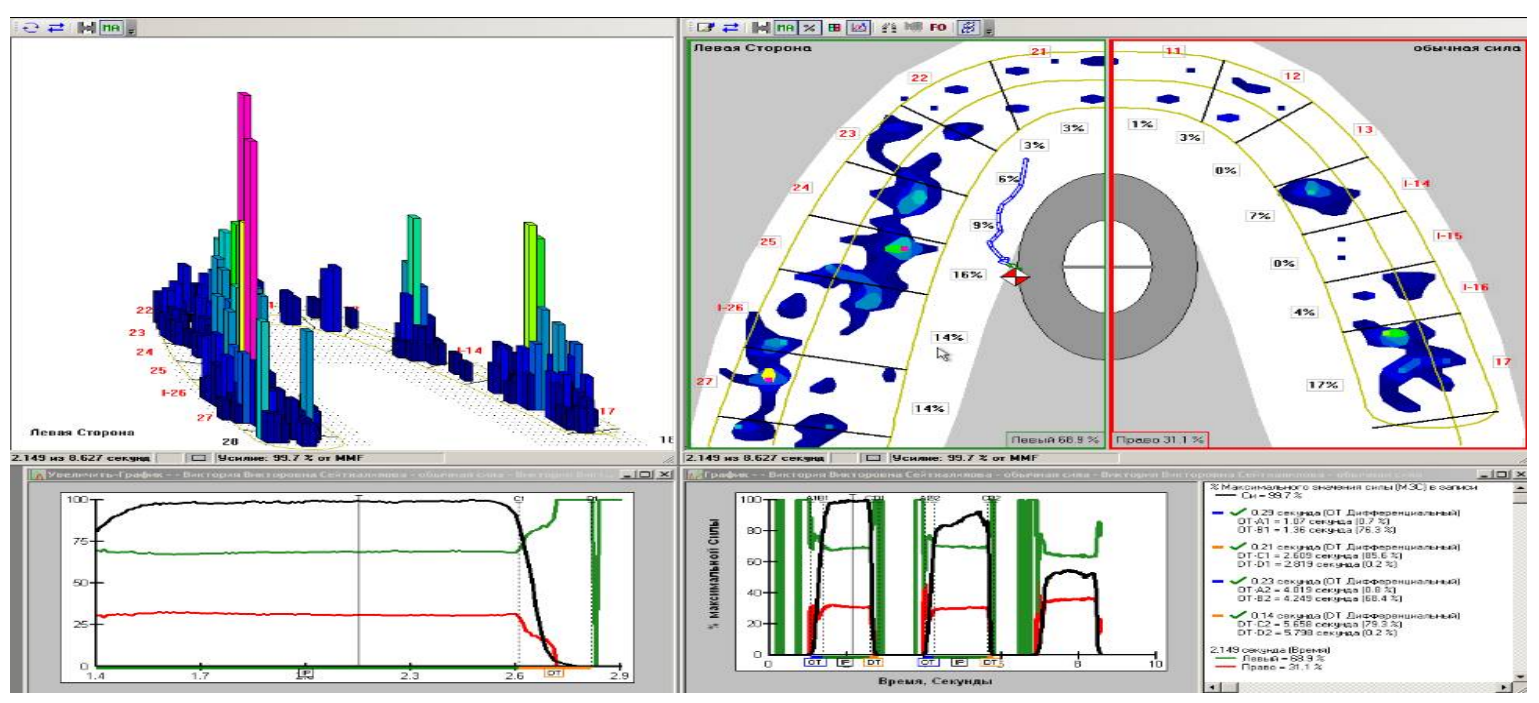

Fig. 3

Table 1

\begin{tabular}{|l|c|}
\hline \multicolumn{1}{|c|}{ Parameter } & Beforetreatment \\
\hline Occlusiontime & Upto $0,2 \mathrm{~ms}$ \\
\hline Disocclusion time & Upto 0,1 ms \\
\hline Balance of the right and left side & $\begin{array}{c}\text { Up to } 45 \%-55 \% \text { (first subgroup) } \\
\text { Up to } 70 \%-30 \% \text { (second subgroup) }\end{array}$ \\
\hline
\end{tabular}

Occlusion time indicators are increased to $0.5-0.7 \mathrm{~ms}$, the disocclusion time is decreased to $0.3 \mathrm{~ms}$, the balance of the right and left side is $40 \%-60 \%$, that is $60 \%-80 \%$ of the allowable limit, which is due to the neurogenic pathology history.

Conclusions. Our treatment demonstrated that the greatest success rate was achieved in patients of the second treatment group.

From the prognostic point of view, the diagnosis and planning of further treatment based on the analysis of occlusive adjustment with T-scan is the most expedient method that allows us to achieve a functional optimum in the future.

\section{References:}


1. Badanin, V. V. (2003). Occlusional splints as the effective method of orthopedic treatment of the functional disorders in TMJ Institut stomatologii, (3), 18-20.

2. Khvatova, V. A. (1998). The diseases of temporo-mandibular joint and the methods of treatment Novoe v stomatologii, (1), 33-48.

3. Davies, J., Gray, R. J. M. (1997). The pattern of splint usage in the management of two common temporomandibular disorders. Br Dental J. P. III: Long-term" follow-up in and assessment of splint therapy in the management of disc displacement with reduction and pain dsfunction syndrome, (8), 46-158.

4. Davies, J., Gray, R. J. M. (1997). The pattern of splint usage in the management of who commen temporomandibular desorders. $\mathrm{Br}$ Dental $\mathrm{J}$. P. II: The stabilization spling in the treatment of pain dysfunction syndrome, (7), 18-186.

5. Dos Santos, J. Jr., Gurklis, M. (1995). Chairside fabrication of occlusal biteplanesplints using visible light cured material. Cranio. (13(2), 6-131.

6. Leib, A. M. (1996). The occlusal bite splint - a noninvasive therapy for occlusal habits and temporomandibular disorders. Compend Contin Educ Dent, (17(11), 4-1081, 1086, 1088.

7. Long, J. H. Jr. (1995). Interocclusal splint designed to reduce tenderness in lateral pterygoid ad other muscles of mastication. J Prosthet Dent. (73(3), 316.

8. Nelson, S. J. Principles of stabilization bite splint therapy. Dent Clin North Am. 1995; 39(2):21—403.

9. Pierce C. J., Weyant R. J., Block H. M. [et al.] (1995). Dental splint prescription patterns: a survey. J Am Dent Assoc. (126(3), 294.

10. Nespryadko, V. P., Tereshchuk, O. G., Shynchukovskyi, I. A., Flis, P. S., Khrol, N. S. (2017). Determination of optimization ways of RE- 
prothesis using various schemes gnathological support of patients at the preparatory stage to the orthopedic correction of patients with occlusive iatrogenic disorders of Tooth-Jaw apparatus. Bioenergetics in medicine and biology, (1(1)2017), 107-115. doi.org/10.26886/25236938.1(1)2017.8

BIOENERGETICS IN MEDICINE AND BIOLOGY. - 2018. - 1(2). - p. 115-126 